\title{
Simplifying laparoscopic running suture line utilizing "Puller" technique: demonstration in laparoscopic myomectomy
}

\author{
Mi-La Kim $\cdot$ Yeon Jean Cho $\cdot$ Joo Myung Kim
}

Received: 20 February 2012/Accepted: 22 October 2012/Published online: 5 January 2013

(C) Springer Science+Business Media New York 2012

\begin{abstract}
Background Laparoscopic myomectomy (LM) has increased recently as treatment options for symptomatic uterine myomas for a patient who wants to preserve her uterus. However, adequate suture of the uterine defect is difficult in LM, even for an experienced surgeon. The most time-consuming step of LM is the suturing procedure. The suture material can tangle easily and disentanglement is time-consuming. We introduce a simple but highly effective instrument named "Puller" for continuous intracorporeal suturing in LM.

Methods After completion of myoma enucleation, the operator sutures the uterine defect with suture material in continuous manner. The tip of "Puller" looks like a hook. During the suture, the first assistant inserts the "Puller" on the suprapubic site and sets the suture material on the hook and pulls it extracorporeally. After one stitch, the operator pulls the suture material intracorporeally, and then the first
\end{abstract}

Electronic supplementary material The online version of this article (doi:10.1007/s00464-012-2689-2) contains supplementary material, which is available to authorized users.

\section{M.-L. Kim}

Department of Obstetrics and Gynecology, CHA Gangnam

Medical Center, CHA University, Seoul, South Korea

\section{Y. J. Cho}

Department of Obstetrics and Gynecology, Dong-A University Medical Center, College of Medicine, Dong-A University, Busan, South Korea

\section{J. M. Kim ( $\square)$}

Department of Obstetrics and Gynecology, Cheil General Hospital and Women's Healthcare Center, Kwandong University College of Medicine, 1-19 Mukjeoung-dong, Jung-gu,

Seoul 100-380, South Korea

e-mail: dr66205@naver.com assistant pulls the sutured portion of the thread extracorporeally with "Puller" and holds the stitch to maintain the adequate tension during the repair.

Results From January 2011 to October 2011, 88 patients who were diagnosed with uterine myoma underwent LM using "Puller" by a single surgeon. The mean diameter of the myoma was $6.8 \pm 2.1 \mathrm{~cm}$, and multiple myomas were observed in 46 cases $(52.3 \%)$. As a result, the mean operation time was $65.0 \pm 22.1 \mathrm{~min}$, the estimated blood loss was $173.9 \pm 179.8 \mathrm{ml}$. Mean weight of removed myoma was $141.5 \pm 105.7 \mathrm{~g}$. Postoperative febrile morbidity (body temperature higher than $37.7^{\circ} \mathrm{C}$ ) was observed in 15 patients $(17 \%)$. However, no patients had conversion to laparotomy and needed blood transfusion. There were no major complications that required reoperation or readmission.

Conclusions Laparoscopic myomectomy can be performed easily and effectively by using the "Puller" technique with standard instruments. Additionally, this "Puller" technique could be adopted in all minimally invasive surgery needed running suture for hemostasis and closure.

Keywords Suturing - Laparoscopy - Myomectomy · Puller

Acknowledgments Special thanks to Dr. Paul I. Lee who invented "Puller" technique.

Conflict of interest Drs. Mi-La Kim, Yeon Jean Cho, and Joo Myung Kim have no conflict of interest or financial ties to disclose. 